\title{
REAL VECTOR BUNDLES AND SPACES WITH FREE INVOLUTIONS
}

\begin{abstract}
BY
ALLAN L. EDELSON( $\left.{ }^{1}\right)$

Abstract. The functor $K R(X)$, defined in [4], is a contravariant functor defined in the category of spaces with involutions. It is shown herein that this functor is classified by equivariant maps into the complex Grassmann manifold, which is given the involution induced by complex conjugation. For the case of free involutions it is shown that the classifying maps can be taken to lie outside the fixed point set of the Grassmann manifold. This fixed point set can be identified with the real Grassmann manifold. It is then shown that, for free involutions, $K R(X)$ is an invariant of the homotopy type of the orbit space $X$ modulo its involution. The multiplicative group of real line bundles, real in the sense of [4], is shown to be classified by equivariant maps into a quadric surface $Q$ in complex projective space. $Q$ carries a free involution and this classification is again valid for spaces with free involutions.
\end{abstract}

I. Introduction. In [4] the definition of Atiyah-real vector bundles was given, and they, together with the associated $K$-theory, were studied. The purpose of this paper is twofold. First we will extend the known classification theorems for complex $K$-theory to the new theory. Second we will obtain new results for the case of Atiyah-real vector bundles over spaces with free involutions. It was anticipated that this functor could be used to obtain a characterization of $M_{k}^{7}$, the differentiable seven sphere defined by Milnor in [1]. This question is studied in $\S I I I$, where $K R\left(M_{k}^{7}\right)$ is computed.

To review the definitions and results of [4], we have that a real space is a topological space $X$ together with a homeomorphism $t: X \rightarrow X$ with $t^{2}=$ identity. We will write $t(x)=\bar{x}$. An Atiyah-real vector bundle over a real space is a complex vector bundle $E \rightarrow X$ which itself is a real space and such that

(1) $\pi: E \rightarrow X$ is equivariant,

(2) the induced map $E_{x} \rightarrow E_{x}$ is conjugate linear.

The Grothendieck group of Atiyah-real vector bundles over the real space $X$ is denoted by $K R(X)$. There is a Bott periodicity theorem and derived cohomology theory.

Define

(1) $R^{p, q}=R^{q}+i R^{p}$,

Received by the editors October 14, 1969.

AMS 1969 subject classifications. Primary 5536, 5730.

Key words and phrases. Atiyah-real vector bundle, conjugate linear isomorphism, equivariant homotopy, line bundle.

${ }^{(1)}$ This paper forms part of the author's doctoral thesis, submitted at the State University of New York at Stony Brook, in June 1968. 
(2) $B^{p, q}=$ unit ball in $R^{p, q}$,

(3) $S^{p, q}=$ unit sphere in $R^{p, q}$,

(4) $K R^{p, q}(X, Y)=K R\left(X \times B^{p, q}, X \times S^{p, q} \cup Y \times B^{p, q}\right)$,

(5) $K R^{p, 0}=K R^{p}, K R^{0, q}=K R^{-q}$.

We then have the following exact sequences:

(I.1) $\cdots K R^{-q}(X) \rightarrow K R^{-q}(Y) \rightarrow K R^{-q+1}(X, Y) \rightarrow \cdots$,

(I.2) $\cdots K R^{p, q}(X) \rightarrow K R^{p, q}(Y) \rightarrow K R^{p, q-1}(X, Y) \rightarrow \cdots(p \geqq 0)$,

(I.3) $\quad 0 \rightarrow K R^{-q}(X) \rightarrow K R^{-q}\left(X \times S^{p, 0}\right) \rightarrow K R^{p+1-q}(X) \rightarrow 0(p \geqq 3)$.

Our first result is the following:

PROPOSITION II.1. There exists a bijective correspondence

$$
\Phi_{n}(X) \rightarrow\left[X, G_{n}\left(C^{\infty}\right)\right]_{\text {equivariant }}
$$

Here $\Phi_{n}(X)$ denotes the isomorphism classes of Atiyah-real vector bundles over the real space $X$. The Grassmannian is given the involution induced by complex conjugation.

If we consider the case of spaces with fixed point free involutions we obtain:

Proposition III.1. If $X$ is a compact space with fixed point free involution $t: X \rightarrow X$, then $K R(X)$ is an invariant of the homotopy type of the orbit space $X / t$.

With this result and the exact sequence I. 3 we obtain

COROLlary. $K R\left(M_{k}^{7}\right) \cong Z$.

These methods can be used to study the group of Atiyah-real line bundles on spaces with free involutions. Some results have been obtained and will appear elsewhere.

II. Classification of real vector bundles. If $V_{k}\left(C^{n}\right)=U(n) / U(n-k)$ denotes the Stiefel variety of orthonormal $k$-frames in $C^{n}$, complex conjugation in $U(n)$ defines an involution of $V_{k}\left(C^{n}\right)$ which sends $\left(v_{1}, \ldots, v_{k}\right)$ to $\left(\bar{v}_{1}, \ldots, \bar{v}_{k}\right)$. Define an involution on the Grassmann manifold $G_{k}\left(C^{n}\right)$ by requiring that the following diagram commute:

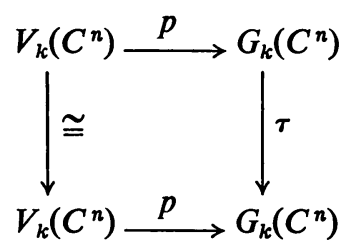

If $V \in G_{k}\left(C^{n}\right)$ has basis $\left\{v_{1}, \ldots, v_{k}\right\}, \tau(V)=\bar{V}$ is the subspace of $C^{n}$ having basis $\left\{\bar{v}_{1}, \ldots, \bar{v}_{k}\right\}$. The classifying bundle $\gamma_{k}^{n}$ is defined to be that subspace of $G_{k}\left(C^{n}\right) \times C^{n}$ consisting of all pairs $(V, x)$ such that $x \in V$. Defining an involution on $G_{k}\left(C^{n}\right) \times C^{n}$ by $\tau \times K$ we clearly have that $\gamma_{k}^{n}$ is invariant under this involution and hence $\tau \times K$ defines an involution on $\gamma_{k}^{n}$ by $(V, x) \rightarrow(\bar{V}, \bar{x})$. Since the projection $\gamma_{k}^{n} \rightarrow G_{k}\left(C^{n}\right)$, 
$(V, x) \rightarrow V$ is equivariant and the map induced on fibres is conjugate linear, we have that $\gamma_{k}^{n}$ is a real vector bundle over the real space $G_{k}\left(C^{n}\right)$. We have the following inclusions:

$$
G_{k}\left(C^{n}\right) \rightarrow G_{k}\left(C^{n+1}\right) \rightarrow \cdots \bigcup_{k \leqq m} G_{k}\left(C^{m}\right)=G_{k}\left(C^{\infty}\right)
$$

which are equivariant and hence an involution is defined on $G_{k}\left(C^{\infty}\right)$. Similarly $\gamma_{k}$ becomes a real vector bundle over the real space $G_{k}\left(C^{\infty}\right)$. If

$$
V=\left\langle v_{1}, \ldots, v_{k}\right\rangle \in G_{k}\left(C^{n}\right)
$$

is a fixed point, it follows that $\bar{v}_{i} \in V$ and hence the vectors $\left\{\left(v_{i}+\bar{v}_{i}\right),-i\left(v_{i}-\bar{v}_{i}\right)\right\}$ are all real, i.e. fixed, and span $V$. Assume then that if $V \in G_{k}\left(C^{n}\right)$ is a fixed point, $V=\left\langle v_{i}, \ldots, v_{k}\right\rangle$ where $v_{i}$ is real for $i=1, \ldots, k$. The map $G_{k}\left(R^{n}\right) \rightarrow G_{k}\left(C^{n}\right)$ defined by $\left\langle x_{1}, \ldots, x_{k}\right\rangle \rightarrow\left\langle x_{1}, \ldots, x_{k}\right\rangle$ is an embedding onto the fixed point set of $G_{k}\left(C^{n}\right)$ for $k \leqq n \leqq \infty$. In particular then taking $k=1$ we have embeddings of $P R^{n}$ and $P R^{\infty}$ onto the fixed point sets of $P C^{n}$ and $P C^{\infty}$ respectively. Now let $X$ and $Y$ be real spaces, $X$ compact and let $\Phi_{n}(X)$ denote the set of isomorphism classes of real $n$-dimensional vector bundles over $X,[X, Y]_{e}$ the set of equivariant homotopy classes of maps $X \rightarrow Y$. As a first result we have

Proposition II.1. There exists a bijective correspondence $\Phi_{n}(X) \rightarrow\left[X, G_{n}\left(C^{\infty}\right)\right]_{e}$.

Proof. By [1, Theorem 2.4] a real vector bundle is locally trivial in the category of real spaces. Let $\xi \stackrel{\pi}{\rightarrow} X$ be a real vector bundle. Then $\forall x \in X \exists$ a neighborhood of $U_{\alpha}$ of $x$ and an isomorphism $h_{\alpha}: \pi^{-1}\left(U_{\alpha}\right) \cong U_{\alpha} \times C^{n}$. If $x=\tau(x)$, then $U_{\alpha}$ can be taken to be a connected open set containing $x$. If $x \neq \tau(x)$ then $U_{\alpha}$ can be taken to be the union of two disjoint open sets $U_{\alpha}=U_{x} \cup U_{\tau(x)}$ where the involution maps $U_{x}$ homeomorphically onto $U_{\tau(x)}$. Let $\left\{U_{i}\right\}_{i=1}^{m}$ be a finite subcovering giving the local product structure, and let $\left\{p_{i}\right\}_{i=1}^{m}$ be a partition of unity subordinate to the given covering so that $\operatorname{cl}\left(p_{i}^{-1}(0,1]\right) \subset U_{i}$. Define a partition of unity by $q_{i}(x)=\frac{1}{2}\left(p_{i}(x)+p_{i}(\tau(x))\right)$. Then $\operatorname{cl}\left(q_{i}^{-1}(0,1]\right) \subset U_{i}$ and $q_{i}(x)=q_{i}(\tau(x))$. Assume then the partition of unity $\left\{p_{i}\right\}$ is equivariant in this sense.

LEMMA II.1. $\exists$ an equivariant map $g_{i}: \pi^{-1}\left(U_{i}\right) \rightarrow C^{2 n}$ which is an isomorphism when restricted to each fibre.

Proof. For $x \in U_{i}$ let $\xi_{x}=\pi^{-1}(x)$ and define $g_{i}: \xi_{x} \rightarrow C^{2 n}$ by the following composition:

$$
\xi_{x} \stackrel{\hat{g}_{i}}{\longrightarrow} R^{2 n} \otimes_{R} C \stackrel{\mu}{\longrightarrow} C^{2 n}
$$

where $\hat{g}_{i}(x, e)=\left(h_{i}(x, e)-h_{i}(\tau(x, e))\right) \otimes 1-i\left(h_{i}(\tau(x, e))-h_{i}(x, e)\right) \otimes i$, and $\mu$ is the $C$-isomorphism $\mu\left[\left(x_{1}, \ldots, x_{2 n}\right) \otimes z\right]=\left(x_{1} z, \ldots, x_{2 n} z\right)$. Then $\hat{g}_{i}$ is a monomorphism 
on each fibre since $\hat{g}_{i}(c)=0 \Rightarrow h_{i}(x, e)+h_{i}(\tau(x, e))=0, \quad h_{i}(x, e)-h_{i}(\tau(x, e))=0$ $\Rightarrow h_{i}(x, e)=0 \Rightarrow e=0$. Also $\hat{g}_{i}$ is $C$-linear since

$$
\begin{aligned}
\hat{g}_{i}(x, i e) & =\left(h_{i}(x, i e)+h_{i}(\tau(x, i e))\right) \otimes 1-1\left(h_{i}(x, i e)-h_{i}(\tau(x, i e))\right) \otimes i \\
& =\left(i h_{i}(x, e)-i h_{i}(\tau(x, e))\right) \otimes 1-i\left(h_{i}(x, e)+i h_{i}(\tau(x, e))\right) \otimes i \\
& =i\left(h_{i}(x, e)-h_{i}(\tau(x, e))\right) \otimes 1+\left(h_{i}(x, e)+h_{i}(\tau(x, e))\right) \otimes i \\
& =i \hat{g}_{i}(x, e) .
\end{aligned}
$$

It is clear that $g_{i}$ is equivariant. Thus $g_{i}: \pi^{-1}\left(U_{i}\right) \rightarrow C^{2 n}$ is the required map.

Now let $\left\{U_{i}\right\}_{i=1}^{m}$ be an open covering of $X$ by real subsets and $g_{i}: \pi^{-1}\left(U_{i}\right) \rightarrow C^{2 n}$ equivariant and a monomorphism on each fibre. Let $\left\{\boldsymbol{P}_{i}\right\}$ be an equivariant partition of unity subordinate to the given covering. Define a map

$$
g: \xi \rightarrow C^{2 n} \oplus \cdots \oplus C^{2 n} \cong C^{2 m n}
$$

by $g(x, e)=\left(p_{1}(x) g_{1}(x, e), \ldots, p_{m}(x) g_{m}(x, e)\right)$. Since $g_{i}\left(\xi_{x}\right)$ and $g_{j}\left(\xi_{x}\right)$ lie in complementary subspaces for $i \neq j$ we have that $g$ is a monomorphism on each fibre. Clearly $g$ is equivariant. $g$ defines a map $\hat{g}: \xi \rightarrow \gamma_{n}^{2 m n}$ by $\hat{g}(x, e)=\left(\left\langle g\left(\xi_{x}\right)\right\rangle, g(x, e)\right)$ and a map of base spaces $\tilde{g}: X \rightarrow G_{n}\left(C^{2 m n}\right)$ be $x \rightarrow\left\langle g\left(\xi_{x}\right)\right\rangle$. We have then proved

LEMMA II.2. If $\xi \stackrel{\pi}{\rightarrow} X$ is a real $n$-dimensional vector bundle, there exists a classifying map $\tilde{g}: X \rightarrow G_{n}\left(C^{2 m n}\right)$ for $m$ sufficiently large, such that $\tilde{g}^{*}\left(\gamma_{n}^{2 m n}\right) \cong \xi$ as real vector bundles.

Now let $j: G_{n}\left(C^{k}\right) \rightarrow G_{n}\left(C^{k+l}\right)$ be the natural equivariant inclusion. The proof of the following is exactly as for the complex case and will be omitted.

LEMMA II.3. If $g: X \rightarrow G_{n}\left(C^{k}\right)$ is a classifying map for the real vector bundle $\xi \stackrel{\pi}{\rightarrow} X$ then the composition $j \circ g: X \rightarrow G_{n}\left(C^{k+l}\right)$ is also a classifying map for $\xi$. From the sequence of equivariant inclusions $G_{n}\left(C^{m}\right) \rightarrow \cdots \rightarrow G_{n}\left(C^{m+k}\right) \rightarrow \cdots$ $\rightarrow G_{n}\left(C^{\infty}\right)$ it follows that given any $\xi \rightarrow X$ there exists a map $f_{\xi}: X \rightarrow G_{n}\left(C^{\infty}\right)$ such that $\xi \cong f_{\xi}^{*}\left(\gamma_{n}\right)$.

LEMMA II.4. Let $f, g: X \rightarrow G_{n}\left(C^{m}\right)$ be two maps such that $f^{*}\left(\gamma_{n}^{m}\right)$ and $g^{*}\left(\gamma_{n}^{m}\right)$ are (real) isomorphic. Let $j: G_{n}\left(C^{m}\right) \rightarrow G_{n}\left(C^{2 m}\right)$ be the natural inclusion. Then $j \circ f \simeq j \circ g$ in the category of real spaces.

Proof. The proof is similar to the complex case. We have a vector bundle $\xi \rightarrow X$ and two vector bundle morphisms $\hat{f}: \xi \rightarrow \gamma_{n}^{m}, \hat{g}: \xi \rightarrow \gamma_{n}^{m}$ inducing maps $f, g$ of the base spaces. $\hat{f}$ and $\hat{g}$ are isomorphisms on each fibre. Define $q: \gamma_{n}^{m} \rightarrow C^{m}$ by $q(v, x)=x$, and $f^{\prime}=q \circ \hat{f}: \xi \rightarrow C^{m}, g^{\prime}=q \circ \hat{g}: \xi \rightarrow C^{m} \cdot f^{\prime}$ and $g^{\prime}$ are monomorphisms on each fibre and are equivariant. We have equivariant homotopies $h_{t}^{e}: C^{n} \rightarrow C^{2 n}$, $h_{t}^{0}: C^{n} \rightarrow C^{2 n}$ defined by

$$
\begin{aligned}
& h_{t}^{e}\left(x_{1}, \ldots, x_{n}\right)=(1-t)\left(x_{1}, x_{2}, \ldots\right)+t\left(x_{1}, 0, x_{2}, 0, \ldots\right), \\
& h_{t}^{0}\left(x_{1}, \ldots, x_{n}\right)=(1-t)\left(x_{1}, x_{2}, \ldots\right)+t\left(0, x_{1}, 0, x_{2}, \ldots\right)
\end{aligned}
$$

and having the following properties: 
(1) $h_{0}^{e}=h_{0}^{0}=$ inclusion: $C^{n} \rightarrow C^{2 n}$,

(2) $h_{1}^{e}\left(C^{n}\right)=C^{2 n} \cap C^{\text {even }}, h_{1}^{0}\left(C^{n}\right)=C^{2 n} \cap C^{\text {odd }}$,

(3) there are vector bundle morphisms

$$
\left(\hat{f}^{e}, f^{e}\right): \gamma_{n}^{m} \rightarrow \gamma_{n}^{2 m}, \quad\left(\hat{f}^{0}, f^{0}\right): \gamma_{n}^{m} \rightarrow \gamma_{n}^{2 m}
$$

such that $q \circ \hat{f}^{e}=h_{1}^{e}, q \hat{f}^{0}=h_{1}^{0}$,

(4) $f^{e}$ and $f^{0}$ are homotopic to inclusion $G_{n}\left(C^{m}\right) \rightarrow G_{n}\left(C^{2 m}\right)$.

A homotopy $f \simeq g$ is then obtained since we have $j \circ f \simeq f^{e} \circ f \simeq f^{0} \circ g \simeq j \circ g$.

We have thus defined a map $\Phi_{n}(X) \rightarrow\left[X, G_{n}\left(C^{\infty}\right)\right]_{e}$ which is clearly onto and is one-to-one by Lemma II.3. This completes the proof of Proposition II.1.

We now consider the case of free involutions, recalling that $G_{k}\left(R^{n}\right)$ can be identified with the fixed point set of $G_{k}\left(C^{n}\right)$.

Proposition II.2. If $X$ is a compact space with fixed point free involution $\exists a$ bijective correspondence $\Phi_{n}(X) \rightarrow\left[X, G_{n}\left(C^{\infty}\right)-G_{n}\left(R^{\infty}\right)\right]_{e}$.

Proof. Notice that $G_{n}\left(C^{\infty}\right)-G_{n}\left(R^{\infty}\right)$ has no fixed points. Thus we are showing that when $X$ has no fixed points the image of $X$ can be pulled off the fixed point set and that homotopies can be taken in the complement of the fixed point set.

LEMMA II.5. Let $X$ be a compact, connected space with fixed point free involution $\tau: X \rightarrow X . \exists$ a finite covering $\left\{U_{i}\right\}_{i=1}^{r}$ of $X$ giving the local product structure, and maps $\left\{g_{i}\right\}_{i=1}^{r}$ having the following properties:

(1) Each $U_{i}$ is the union of two disjoint open sets, $U_{i}=U_{x_{i}} \cup U_{\tau\left(x_{i}\right)}$ with $U_{x_{i}} \cap U_{\tau\left(x_{i}\right)}=\varnothing, \tau\left(U_{x_{i}}\right)=U_{\tau\left(x_{i}\right)}$.

(2) $g_{i}: \pi^{-1}\left(U_{i}\right) \rightarrow C^{2 n}$ is equivariant and a monomorphism on each fibre.

(3) If $x \in U_{i}$, so that $\tau(x) \in U_{i}$, then $g_{i}\left(\xi_{x}\right) \neq g_{i}\left(\xi_{\tau(x)}\right)$ as $n$-dimensional subspaces of $C^{2 n}$.

Proof. Let $\left\{U_{i}\right\}_{i=1}^{r},\left\{g_{i}\right\}_{i=1}^{r}$ be constructed as in the proof of Lemma II.1. Then since $X$ has no fixed points the covering $\left\{U_{i}\right\}$ can be taken to be the union of two open sets, disjoint, and conjugate under $\tau$. We have the map $g_{i}: \pi^{-1}\left(U_{i}\right) \rightarrow C^{2 n}$ which is equivariant and a monomorphism on each fibre. Then $g_{i}$ induces a map $\varphi_{i}: \pi^{-1}\left(U_{i}\right) \rightarrow \gamma_{n}^{2 n}$ by $\varphi_{i}(x, e)=\left(\left\langle g_{i}\left(\xi_{x}\right)\right\rangle, g_{i}(x, e)\right)$ and hence a classifying map $\tilde{\varphi}_{i}: U_{i} \rightarrow G_{n}\left(C^{2 n}\right)$ defined by $x \rightarrow\left\langle g_{i}\left(\xi_{x}\right)\right\rangle$. Since $\left.\xi\right|_{U_{i}}$ is trivial in the category of real spaces by [1, Theorem 2.4] the map $\tilde{\varphi}_{i}$ is equivariantly homotopic to a map $\tilde{\varphi}_{i}^{\prime}: U_{i} \rightarrow G_{n}\left(C^{2 n}\right)$ which sends the components $U_{x_{i}}$ and $U_{\tau\left(x_{i}\right)}$ to distinct conjugate points of $G_{n}\left(C^{2 n}\right)$. Thus $g_{i}$ is homotopic to a map $g_{i}^{\prime}: \pi^{-1}\left(U_{i}\right) \rightarrow C^{2 n}$ such that $g_{i}^{\prime}\left(\xi_{x}\right) \neq g_{i}^{\prime}\left(\xi_{\tau(x)}\right)$ as $n$-dimensional subspaces of $C^{2 n}$. This proves Lemma II.4.

Again choose an equivariant partition of unity $\left\{p_{i}\right\}_{i=1}^{r}$ subordinate to the given covering. Define $g: \xi \rightarrow C^{2 m n}$ as before by $g(x, e)=\left(p_{1}(x) g_{1}(x, e), \ldots, p_{m}(x) g_{m}(x, e)\right)$, where the $g_{i}$ are taken to satisfy the conditions of Lemma II.4. As before $g$ is equivariant and a monomorphism on each fibre. Since the involution on $X$ is free we now must show that $g\left(\xi_{x}\right) \neq g\left(\xi_{\tau(x)}\right)$ as $n$-dimensional subspaces of $C^{2 m n}$. Let 
$\left\{e_{i}\right\}_{i=1}^{n}$ be a basis for $\xi_{x}$, so that $\left\{\tau\left(x_{i}\right)\right\}$ is a basis for $\xi_{\tau(x)}$. Then $\left\{g\left(x, e_{i}\right)\right\}$ $=\left\{\left(\left(p_{1}(x) g_{1}\left(x, e_{i}\right), \ldots, p_{m}(x) g_{m}\left(x, e_{i}\right)\right)\right\}\right.$ is a basis for $g\left(\xi_{x}\right)$. If $g\left(\xi_{x}\right)=g\left(\xi_{\tau(x)}\right)$ then $\left(p_{1}(x) g_{1}\left(\tau\left(x, e_{i}\right)\right), \ldots, p_{m}(x) g_{m}\left(\tau\left(x, e_{i}\right)\right)\right)=\sum_{j=1}^{n} \lambda_{i j}\left(p_{1}(x) g_{1}\left(x, e_{j}\right), \ldots, p_{m}(x) g_{m}\left(x, e_{j}\right)\right)$.

Thus $p_{k}(x) g_{k}\left(\tau\left(x, e_{i}\right)\right)=\sum_{j=1}^{n} \lambda_{i j}\left(p_{k}(x) g_{k}\left(e, e_{j}\right)\right), i=1,2, \ldots, n$, and $k=1, \ldots, m$. This contradicts the requirements that $g_{k}\left(\xi_{x}\right) \neq g_{k}\left(\xi_{\tau(x)}\right)$ for $x \in U_{k}$ and the lemma is proved.

We then have that $g: \xi \rightarrow C^{2 m n}$ defines a map $\hat{g}(x, e)=\left(\left\langle g\left(\xi_{x}\right)\right\rangle, g(x, e)\right)$ and a map of base spaces $X \rightarrow G_{n}\left(C^{2 m n}\right)$ by $x \rightarrow\left\langle g\left(\xi_{x}\right)\right\rangle$. Following by the inclusion $G_{n}\left(C^{2 m n}\right) \rightarrow G_{n}\left(C^{\infty}\right)$ we have the required classifying map. Since $g\left(\xi_{x}\right) \neq g\left(\xi_{\tau(x)}\right)$ the map $X \rightarrow G_{n}\left(C^{\infty}\right)$ lies in the complement of the fixed point set and hence is a $\operatorname{map} X \rightarrow G_{n}\left(C^{\infty}\right)-G_{n}\left(R^{\infty}\right)$.

Proposition II.2 will be proven when we have shown that homotopies between two classifying maps can be taken to lie in the complement of the fixed point set.

LEMMA II.6. If $f, g: X \rightarrow G_{n}\left(C^{m}\right)-G_{n}\left(R^{m}\right)$ are such that $f^{*}\left(\gamma_{n}^{m}\right) \cong g^{*}\left(\gamma_{n}^{m}\right)$ as real vector bundles, i.e. the pullbacks of $\gamma_{n}^{m} \mid G_{n}\left(C^{m}\right)-G_{n}\left(R^{m}\right)$ are isomorphic, then there is a homotopy $j \circ f \simeq j \circ g: X \rightarrow G_{n}\left(C^{2 m}\right)-G_{n}\left(R^{2 m}\right)$.

Proof. In the proof of Lemma II.3 equivariant homotopies $h_{t}^{e}$ and $h_{t}^{0}: C^{m} \rightarrow C^{2 m}$ were defined. We have $h_{t}^{e}$ a linear monomorphism with matrix

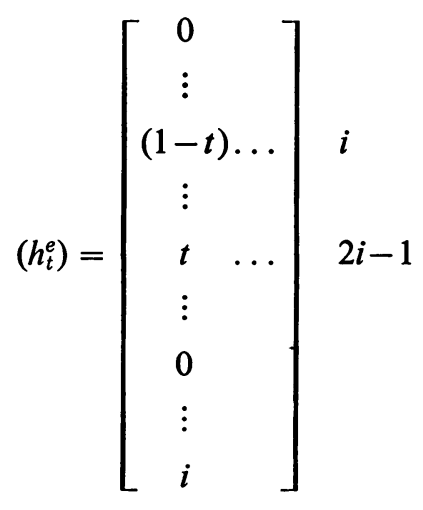

and a similar matrix for $\left(h_{t}^{0}\right)$. Since these matrices have real entries the transformations $h_{t}^{e}$ and $h_{t}^{0}$ commute with complex conjugation and hence are equivariant. Thus the maps $\hat{f}^{e}$ and $\hat{f}^{0}$ are equivariant so that $f_{t}^{e} \circ f(X) \subset G_{n}\left(C^{m}\right)-G_{n}\left(R^{m}\right)$ and $f_{t}^{0} \circ g(X) \subset G_{n}\left(C^{m}\right)-G_{n}\left(R^{m}\right)$ provided $f(X), g(X) \subset G_{n}\left(C^{m}\right)-G_{n}\left(R^{m}\right)$. It follows then that there is a homotopy $f \simeq g: X \rightarrow G_{n}\left(C^{2 m}\right)-G_{n}\left(R^{2 m}\right)$ and Lemma II.5 is proved. This completes the proof of Proposition II.2.

III. Fixed point free involutions. We assume $X$ is compact, connected, simply connected and that $\tau: X \rightarrow X$ acts freely. By §II any real $n$-dimensional vector bundle $\xi$ over $X$ corresponds to a map $f_{\xi}: X \rightarrow G_{n}\left(C^{m}\right)-G_{n}\left(R^{m}\right)$ for sufficiently large $m$. If $W \in G_{k}\left(C^{n}\right)-G_{k}\left(R^{n}\right)$ and $W^{\prime} \in G_{l}\left(C^{m}\right)$, the sum $W \oplus W^{\prime}$ is not left 
fixed by complex conjugation in $G_{k+\imath}\left(C^{n+m}\right)$ since the images of $W$ and $W^{\prime}$ lie in complementary subspaces of $C^{n+m}$. Thus $W \oplus W^{\prime} \in G_{k+l}\left(C^{n+m}\right)-G_{k+l}\left(R^{n+m}\right)$. This is true provided $k>0$. The following lemma is proved as for the complex case and the proof is omitted:

LEMMA III.1. If $f_{\xi}: X \rightarrow G_{k}\left(C^{n}\right)-G_{k}\left(R^{n}\right)$ and $g_{n}: X \rightarrow G_{l}\left(C^{m}\right)-G_{l}\left(R^{m}\right)$ are classifying maps for $\xi$ and $\eta$ respectively, then the composition

$$
d\left(f_{\xi} \times g_{n}\right) \Delta: X \rightarrow G_{k+l}\left(C^{n+m}\right)-G_{k+l}\left(R^{n+m}\right)
$$

is a classifying map for $\xi \oplus \eta$. Here $\Delta: X \rightarrow X \times X$ is the diagonal map and $d$ is induced by the map $C^{n} \times C^{m} \rightarrow C^{n+m}$ sending

$$
\left(z_{1}, \ldots, z_{n}\right),\left(w_{1}, \ldots, w_{m}\right) \rightarrow\left(z_{1}, \ldots, z_{n}, w_{1}, \ldots, w_{m}\right) .
$$

Since the trivial bundle $\theta_{m}$ can be classified by the unique map $X \rightarrow G_{m}\left(C^{m}\right)$ we have the following

LEMMA III.2. If $f_{\xi}: X \rightarrow G_{k}\left(C^{n}\right)-G_{k}\left(R^{n}\right), k>0$, is a classifying map for the real bundle $\xi \rightarrow X$, then $j \circ f_{\xi}: X \rightarrow G_{k+m}\left(C^{n+m}\right)-G_{k+m}\left(R^{n+m}\right)$ is a classifying map for $\xi \oplus \theta_{m}$. Here $j: G_{k}\left(C^{n}\right)-G_{k}\left(R^{n}\right) \rightarrow G_{k+m}\left(C^{n+m}\right)-G_{k+m}\left(R^{n+m}\right)$ is the natural inclusion.

If $B U=\bigcup_{1 \leqq n} G_{n}\left(C^{2 n}\right)$ then $B O=\bigcup_{1 \leqq n} G_{n}\left(R^{2 n}\right)$ can be identified with the fixed point set of $B U$ under the involution induced by complex conjugation. Every element of $K R(X)$ can be written as $[\eta]-\left[\theta_{n}\right]$ where $\eta$ is an $n$-dimensional real vector bundle over $X$. If $\eta$ is an $n$-dimensional real bundle over $X$ with classifying map $f_{n}: X \rightarrow G_{n}\left(C^{m}\right)-G_{n}\left(R^{m}\right)$ there is a bundle $\eta^{\prime} \rightarrow X$ with classifying map $f_{\eta^{\prime}}: X \rightarrow G_{k}\left(C^{l}\right)-G_{k}\left(R^{l}\right)$ such that $\eta \oplus \eta^{\prime} \cong \theta_{n+k}$ has classifying map

$$
f: X \rightarrow G_{k+n}\left(C^{m+l}\right)-G_{k+n}\left(R^{m+l}\right) .
$$

Define $f_{0}: C^{\infty} \rightarrow C^{\infty}$ by $f_{0}\left(e_{i}\right)=e_{2 i}$ and $f_{e}: C^{\infty} \rightarrow C^{\infty}$ by $f_{e}\left(e_{i}\right)=e_{2 i}$. These maps give rise to $\psi_{n}: G_{n}\left(C^{2 n}\right) \times G_{n}\left(C^{2 n}\right) \rightarrow G_{2 n}\left(C^{4 n}\right)$, where $\psi_{n}\left(W, W^{\prime}\right)=f_{0}(W)+f_{e}\left(W^{\prime}\right)$. Since $f_{0}(W)$ and $f_{e}\left(W^{\prime}\right)$ lie in complementary subspaces of $C^{4 n}$ there is an induced map $\psi_{n}: G_{n}\left(C^{2 n}\right)-G_{n}\left(R^{2 n}\right) \times G_{n}\left(C^{2 n}\right)-G_{n}\left(R^{2 n}\right) \rightarrow G_{2 n}\left(C^{4 n}\right)-G_{2 n}\left(R^{4 n}\right)$. The product $B U \times B U \rightarrow B U$ is induced by $\psi_{n}$. We have the following commutative diagram where the vertical maps are inclusions:

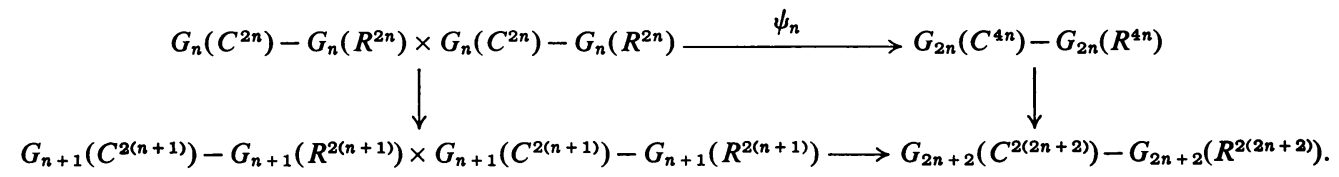

Thus there is a product induced

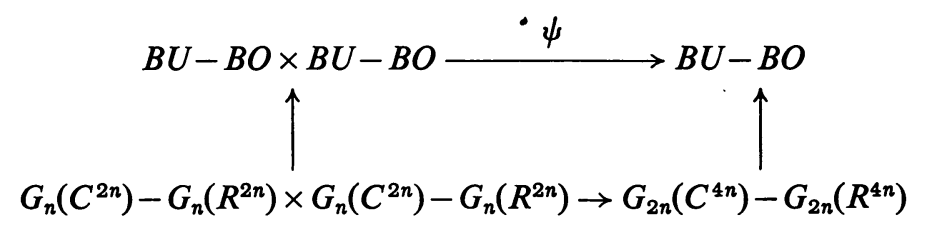


so that the above diagram commutes. If $f: X \rightarrow B U-B O$ we can assume that $f: X \rightarrow G_{n}\left(C^{2 n}\right)-G_{n}\left(R^{2 n}\right)$ for sufficiently large $n$. Define

$$
\theta:[X, B U-B O]_{e} \rightarrow K R(X)
$$

by $\theta[f]=f^{*}\left(\gamma_{n}^{2 n}\right)-\theta_{n}$.

LEMMA III.3. $\theta$ is an isomorphism of groups.

Proof. $\theta[f]=f^{*}\left(\gamma_{n}^{2 n}\right)-\theta_{n}$, and $\theta[g]=g^{*}\left(\gamma_{n}^{2 n}\right)-\theta_{n}$. There is an equivariant homotopy

$$
\psi_{n} \simeq d: G_{n}\left(C^{2 n}\right)-G_{n}\left(R^{2 n}\right) \times G_{n}\left(C^{2 n}\right)-G_{n}\left(G^{2 n}\right) \rightarrow G_{2 n}\left(C^{4 n}\right)-G_{2 n}\left(R^{4 n}\right) .
$$

Thus

$$
\begin{aligned}
\left(\psi_{n}(f \times g) \Delta\right)^{*}\left(\gamma_{2 n}^{4 n}\right) & =(d(f \times g) \Delta)^{*}\left(\gamma_{2 n}^{4 n}\right) \\
& =\Delta^{*}\left(f^{*}\left(\gamma_{n}^{2 n}\right) \times g^{*}\left(\gamma_{n}^{2 n}\right)\right)=f^{*}\left(\gamma_{n}^{2 n}\right) \oplus g^{*}\left(\gamma_{n}^{2 n}\right),
\end{aligned}
$$

and so

$$
\begin{aligned}
\theta\left[\psi_{n}(f \times g) \Delta\right] & =f^{*}\left(\gamma_{n}^{2 n}\right) \oplus g^{*}\left(\gamma_{n}^{2 n}\right)-\theta_{2 n} \\
& =\left(f^{*}\left(\gamma_{n}^{2 n}\right)-\theta_{n}\right)+\left(g^{*}\left(\gamma_{n}^{2 n}\right)-\theta_{n}\right)=\theta[f]+\theta[g] .
\end{aligned}
$$

Now let $\hat{f}: X \rightarrow B U-B O$ be an equivariant map. There is then an induced map $f: X / \tau \rightarrow B U-B O / \tau$ defined by $f[x]=\left[\pi_{2} \circ \hat{f}(x)\right]$ where $\pi_{2}$ is the projection. We have the following commutative diagram:

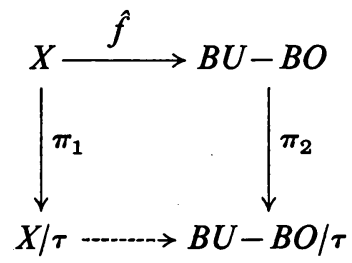

Clearly an equivariant homotopy $\hat{f} \simeq \hat{g}$ defines a homotopy $f \simeq g$ and thus there is a function $\varphi:[X, B U-B O]_{e} \rightarrow[X, \tau, B U-B O / \tau]$. We now require information about the fibration $B U-B O \stackrel{\pi_{2}}{\rightarrow} B U-B O / \tau$.

LEMMA III.4. $B U-B O$ is simply connected.

Proof. For dimensional reasons $G_{n}\left(C^{2 n}\right)-G_{n}\left(R^{2 n}\right)$ is arcwise connected for $n \geqq 2$. It is obtained from $G_{n-1}\left(C^{2 n-2}\right)-G_{n-1}\left(R^{2 n-2}\right)$ by attaching cells $e^{2 k}-e^{k}$, where $k>1$. Each $e^{2 k}-e^{k}$ is a $2 k$-dimensional cell with a $k$-dimensional cell removed. We refer to [2] for a complete description of the cell structure of $G_{n}\left(C^{2 n}\right)$. Thus the quotient space

$$
G_{n}\left(C^{2 n}\right)-G_{n}\left(R^{2 n}\right) / G_{n-1}\left(C^{2 n-2}\right)-G_{n-1}\left(R^{2 n-2}\right)
$$

is a wedge of spheres $S^{2 k}-D^{k}, k>1$. Each $S^{2 k}-D^{k}$ is a $2 k$-sphere with a $k$-disc removed. Since $D^{k}$ is contractable in $S^{2 k}$, it follows that any loop in $G_{n}\left(C^{2 n}\right)$ 
$-G_{n}\left(R^{2 n}\right)$ can be deformed into $G_{1}\left(C^{2}\right)-G_{1}\left(R^{2}\right)$, which is a disjoint union of two 2-discs. Hence every loop is null-homotopic. Thus each $G_{n}\left(C^{2 n}\right)-G_{n}\left(R^{2 n}\right)$ is simply connected. By Proposition 4.3 of [3] the inclusion $G_{n}\left(C^{2 n}\right)-G_{n}\left(R^{2 n}\right) \rightarrow B U-B O$ induces an isomorphism of fundamental groups. Hence $(B U-B O)$ is simply connected. Let $g: B U-B O / \tau \rightarrow R P^{\infty}$ be a classifying map for the principal $z_{2^{-}}$ bundle $B U-B O \stackrel{\pi_{2}}{\rightarrow} B U-B O / \tau$. By Lemma III.4 $\pi_{1}(B U-B O)=0$; the same is true of $X$ by assumption. Thus $B U-B O$ and $X$ are the universal covering spaces of their orbit spaces $B U-B O / \tau$ and $X / \tau$. It follows then that the corresponding line bundles $g^{*}(\gamma(1))$ and $(g \circ f)^{*}(\gamma(1))$ have nonzero Stiefel Whitney classes $w_{1} \neq 0$. Here $\gamma(1)$ is the canonical line bundle $\gamma(1) \rightarrow R P(1)=B O(1)$. By naturality of the Stiefel Whitney classes and the fact that $w_{1}(\gamma(1)) \neq 0$ we have $f^{*}\left(w_{1}(\gamma(1))\right)$ $=w_{1}\left(f^{*}(\gamma(1))\right)$ and $f^{*} g^{*}\left(w_{1}(\gamma(1))\right)=w_{1}\left((g \circ f)^{*}(\gamma(1))\right)$. From the exact homotopy sequence of the fibrations $X \stackrel{\pi_{1}}{\rightarrow} X / \tau$ and $B U-B O \stackrel{\pi_{2}}{\rightarrow} B U-B O / \tau$ we have that $\left.\pi_{1}(X / \tau) \cong \pi_{1}(B U-B O / \tau) \cong \pi_{1}(\overrightarrow{R P})^{\infty}\right) \cong z_{2}$ Hence

and

$$
g^{*}: H^{1}\left(R P^{\infty} ; z_{2}\right) \stackrel{\cong}{\longrightarrow} H^{1}\left(B U-B O / \tau ; z_{2}\right)
$$

$$
f^{*}: H^{1}\left(B U-B O / \tau ; z_{2}\right) \stackrel{\cong}{\longrightarrow} H^{1}\left(X / \tau ; z_{2}\right) .
$$

Let $[X / \tau, B U-B O / \tau]^{\prime}$ denote the set of homotopy classes of maps

$$
f: X / \tau \rightarrow B U-B O / \tau
$$

such that $f^{*}: H^{1}\left(B U-B O / \tau ; z_{2}\right) \cong H^{1}\left(X / \tau ; z_{2}\right)$. Then we have

$$
\varphi:[X, B U-B O]_{e} \rightarrow[X / \tau, B U-B O / \tau]^{\prime} .
$$

Now let $f \in[X / \tau, B U-B O / \tau]^{\prime}$. Then the composition $f \circ \pi_{1}: X \rightarrow B U-B O / \tau$ lifts to a map $\hat{f}: X \rightarrow B U-B O$ such that $f \circ \pi_{1}=\pi_{2} \circ \hat{f} . f^{*} \neq 0$ implies that $\hat{f}$ is a bundle map and one-one on fibres. Hence $\hat{f}$ is equivariant. The map

$$
\psi:[X / \tau, B U-B O / \tau]^{\prime} \rightarrow[X, B U-B O]_{e}
$$

thus defined is clearly an inverse to $\varphi$, and we have that $\varphi$ defines a bijective correspondence $[X / \tau, B U-B O / \tau]^{\prime} \leftrightarrow[X, B U-B O]_{e}$.

Proposition III.1. If $X$ is connected and simply connected with fixed point free involution $\tau: X \rightarrow X$ then $K R(X)$ is an invariant of the homotopy type of the orbit space $X / \tau$.

Proof. Let $X$ and $Y$ be as above and $h: X / \tau$ a homotopy equivalence. Then $h$ defines a bijective correspondence $h^{*}:[Y / \tau, B U-B O / \tau]^{\prime} \rightarrow[X / \tau, B U-B O / \tau]^{\prime}$. We have the following sequence of bijections:

$$
\begin{aligned}
K R^{\sim}(X) \stackrel{\theta_{x}}{\longleftarrow}[X, B U-B O]_{e} \stackrel{\varphi_{x}}{\longrightarrow}[X / \tau, B U-B O / \tau]^{\prime} \\
\stackrel{h^{*}}{\longleftarrow}[Y / \tau, B U-B O / \tau]^{\prime} \stackrel{\varphi_{y}}{\longleftarrow}[Y, B U-B O]_{e} \stackrel{\theta_{y}}{\longrightarrow} K R^{\sim}(Y)
\end{aligned}
$$


where $\theta_{x}$ and $\theta_{y}$ are group isomorphisms. Let $\alpha: K R^{\sim}(X) \rightarrow K R^{\sim}(Y)$ be the composition of the above bijections.

LEMMA III.5. $\alpha$ is a group isomorphism.

Proof. Let $[f],[g] \in[X, B U-B O]_{e}$. Then it suffices to prove $\alpha([f]+[g])$ $=\alpha[f]+\alpha[g]$ where by an abuse of notation we let $\alpha$ denote the map $[X, B U-B O]_{e} \rightarrow[Y, B U-B O]_{e}$. Since $X$ is compact we can assume

$$
f, g: X \rightarrow G_{n}\left(C^{2 n}\right)-G_{n}\left(R^{2 n}\right) .
$$

If $\hat{h}$ is a lifting of $h$ to $Y$, then we have the following:

$$
\begin{aligned}
& \alpha([f]+[g])=\Psi_{n} \circ(f \times g) \circ \Delta_{x} \circ \hat{h}, \\
& \alpha[f]+\alpha[g]=\Psi_{n} \circ(f \times g) \circ(\hat{h} \times \hat{h}) \circ \Delta_{y} .
\end{aligned}
$$

Here $\Delta$ denotes the diagonal map. These maps are in fact equal, which proves Lemma III.5 and hence completes the proof of Proposition III.1.

Let $M_{k}^{7}$ denote the exotic seven-sphere defined in [1]. Then $M_{k}^{7}$ is homeomorphic to $S^{7}$ and carries a fixed point free involution $\tau: M_{k}^{7} \rightarrow M_{k}^{7}$. By [1] there is a homotopy equivalence $h: M_{k}^{7} / \tau \rightarrow P^{7}$. Using the notation of [4] $S^{p, 0}$ denotes the $p-1$ sphere with antipodal involution, and there is the following short exact sequence which splits naturally:

$$
0 \rightarrow K R^{-q}(X) \rightarrow K R^{-q}\left(X \times S^{p, 0}\right) \rightarrow K R^{p+1-q}(X) \rightarrow 0 \text { for } p \geqq 3, q \geqq 0 .
$$

Taking $X=$ point, $q=0, p=8$, we have

$$
K R^{-q}(X) \cong K R(p t) \cong K O(p t) \cong Z, \quad K R^{p+1-q}(X)=K R^{9}(p t) .
$$

By [4] $K R^{9}(p t) \cong K R^{-7}(p t) \cong \tilde{K} O\left(S^{7}\right)=0$. Thus we have $K R\left(S^{8,0}\right) \cong Z \oplus 0=Z$. By Theorem III.1 there is an isomorphism $\alpha: K R\left(M_{k}^{7}\right) \cong K R\left(S^{8,0}\right)$.

Corollary. $K R\left(M_{k}^{7}\right) \cong Z$.

\section{BIBLIOGRAPHY}

1. J. Milnor, On manifolds homeomorphic to the 7-sphere, Ann. of Math. (2) 64 (1956), 399405. MR 18, 498.

2. - Lectures on characteristic classes, Princeton University, 1957.

3. D. Husemoller, Fibre bundles, McGraw-Hill, New York, 1966. MR 37 \#4821.

4. M. F. Atiyah, K-theory and reality, Quart. J. Math. Oxford Ser. (2) 17 (1966), 367-386. MR 34 \#6756.

5. — K-theory, Lectures, Harvard University (1964), Benjamin, New York, 1967. MR 36 \#7130.

6. P. Landweber, Conjugations on complex manifolds and equivariant homotopy of $M U$, Bull. Amer. Math. Soc. 74 (1968), 271-274.

7. N. Steenrod, Topology of fibre bundles, Princeton Math. Series, vol. 14, Princeton Univ. Press, Princeton, N. J., 1951. MR 12, 522.

UNIVERSITY OF CALIFoRNiA, Davis, California 95616 